\title{
A High Performance Point Based Image Alignment Approach Using an Artificial Immune System
}

\author{
Osama A. Omer, Member, IACSIT and Mohamed Abdel-Nasser
}

\begin{abstract}
In this paper, a high performance image alignment approach is presented. This approach is classified as a point based alignment approach. An artificial immune system (AIS) with a modified mutation formula is used to find the correspondence points between the reference and the input images. After the correspondence is found, the least mean squares technique (LMS) is used to determine the transformation which is used to align the two images. This approach doesn't require any additional refinement or features detector as some others approaches required. To demonstrate the effectiveness of proposed algorithm, it compared with two state-of-the-art algorithms for different data sets.
\end{abstract}

Index Terms-Artificial immune system, mutation, correspondence, least squares minimization.

\section{INTRODUCTION}

Image registration is the process of transforming different sets of images into one coordinate system, and it is widely used in various applications. Images may be photographs, images from different sensors, from different times, or from different viewpoints. Many common minimization strategies have been applied to image registration problems, such as exhaustive search, gradient descent [1], simulated annealing and genetic algorithms (GA) [2].

There are two basic steps of image registration process. First is to determine the similarity function. An image similarity measure quantifies the degree of similarity between intensity patterns in two images. The choice of an image similarity measure depends on the modality of the images to be registered. Common examples of image similarity measures include cross-correlation [3], mutual information and sum of squared intensity differences. The second step is to solve the registration model.

Traditional optimization methods are easy to fail being trapped in one of the local minima so a global optimization technique is often required. Janko et al. [4] presented a successful application of GA to the registration of uncelebrated optimal images. F. L. Seixas et. al. [5] proposed image registration using genetic algorithm, in this work the point matching problem was addressed employing a method based on nearest neighbour however, this paper not evaluating the GA considering a real-world application. K. K. Delibasis et. al [6] proposed automatic point correspondence using an artificial immune system optimization technique for medical image registration, in this paper a modified mutation

Manuscript received August 17, 2012; revised November 18, 2012

The authors are with the Department of Electrical Engineering, Aswan University, Aswan, Egypt (e-mail: omer.osama@gmail.com, egnaser@gmail.com). formula based on an uniform distribution was used. F. Ye et. al [7] proposed two step image registration by artificial immune system and chamfer matching, in this paper the artificial immune system has been used to find an initial transformation where the edge distance used as a fitness function then an area based method has been used to refine the transformation estimation.

The artificial immune systems are used for function optimization, the clonal selection and affinity mutation principles are used to explain how the immune systems perform the optimization process. There are many artificial immune systems were published in the context. An immune algorithm, named CLONALG, was developed to perform pattern recognition and optimization. De Castro and J. Timmis proposed opti-aiNet for Multimodal Function Optimization [8]. This algorithm is used in our registration approach. The mutation formula proposed at [8] was:

$$
c^{-}=c+\alpha \cdot N(0,1), \alpha=(1 / \beta) \cdot \exp \left(-f^{*}\right)
$$

Where $C^{-}$is the mutated component, $\mathrm{c}$ is the old component, $\mathrm{N}(0,1)$ is a random variable with Gaussian distribution with zero mean and unity standard deviation, $\alpha$ is the mutation ratio, $\mathrm{f}^{*}$ is the fitness for an individual (solution) normalized between the interval $[0,1], \beta$ is a parameter that controls the decay of the inverse exponential function.

In [6] instead of a Gaussian random variable an uniform random variable is used, however we study the distribution of warping parameter as will be shown in the next section,we found that the parameter distribution were not uniform and it can be considered as a Gaussian random variable.

Therefore, we propose to perform image registration using AIS by incorporating modified mutation formula as it will be shown in section 3 . The AIS randomly selects some points in the reference image then it search for its correspondence in the input image, after the correspondence is found the least mean square technique is used to find the transformation that align the reference and input images. This approach doesn't require a features detector as it usual in all point based image registration algorithms in addition to it doesn't need any refinement as the approach proposed at [7].

\section{THE Distribution OF WARP PARAMETERS}

The distribution of the warp parameters have been studied between many pairs of images. In this study; the first image of the car sequence is used as a reference image and the reset 63 images of the car sequence used as input images, the additive lucas -kanade (LK) registration algorithm is used to 
study the warp parameters. The distributions of the six affine parameters are shown in Fig. 1, the scaling parameter along $\mathrm{x}$-axis $\mathrm{p} 1$ is shown in (a), p4 the scaling parameter a long $\mathrm{y}$-axis is shown in (b), the shearing parameter along $\mathrm{x}$-axis $\mathrm{p} 2$ is shown in (c), the shearing parameter along $y$-axis $\mathrm{p} 3$ is shown in (d), p5 is the translation along $x$-axis shown in (e), finally the translation along $y$-axis p6 is shown in (f). It is easy to notice that the parameters distribution is not uniform as introduced in [6] and it can be considered as Gaussian distribution. Based on this study in the mutation part we will modify Eq. (1) to be suitable for the purpose of image registration.

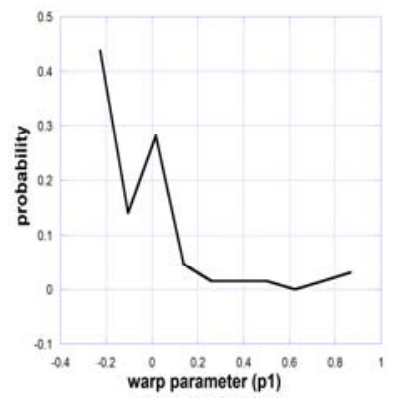

(a)

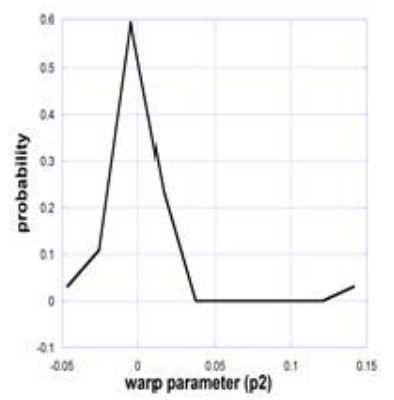

(c)

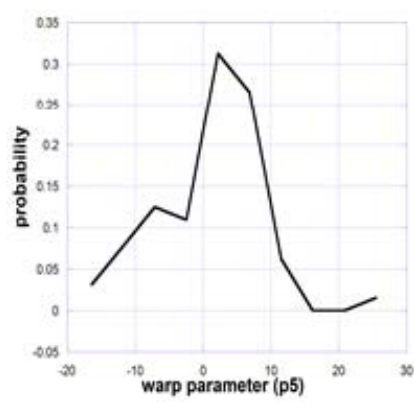

(d)

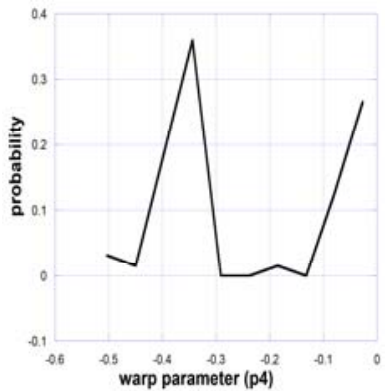

(b)

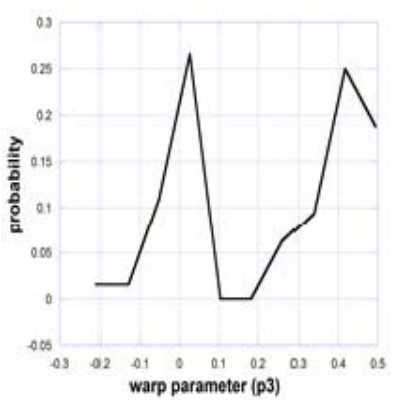

(c)

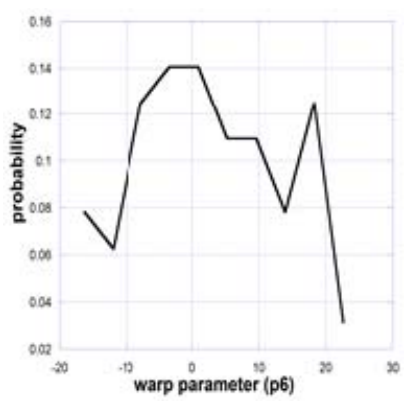

(e)
Fig. 1. The affine parameters distribution, (a) p1, (b) p4, (c) p2, (d) p3, (e) p5, (f) $\mathrm{p} 6$.

\section{The Proposed REgistration APPROACH}

The diagram describing the proposed approach is shown in Fig. 2, the correspondence using an AIS consists of the following stages:

1) The population is initialized in the search range with $n_{\text {int }}$ cell. The population arrangement is described in Fig. 3, where each row represent a cell, each cell consists of six components (x,y,dx,dy,s, $\theta)$, these components represent the reference image point location $(x, y)$.

2) The similarity transformation is used as a registration model, Eq. (2) described this transformation where dx is the translation along $\mathrm{x}$-axis, $\mathrm{dy}$ is the translation along $\mathrm{y}$-axis, $\mathrm{s}$ is the scaling factor and $\theta$ is the rotation in degrees.

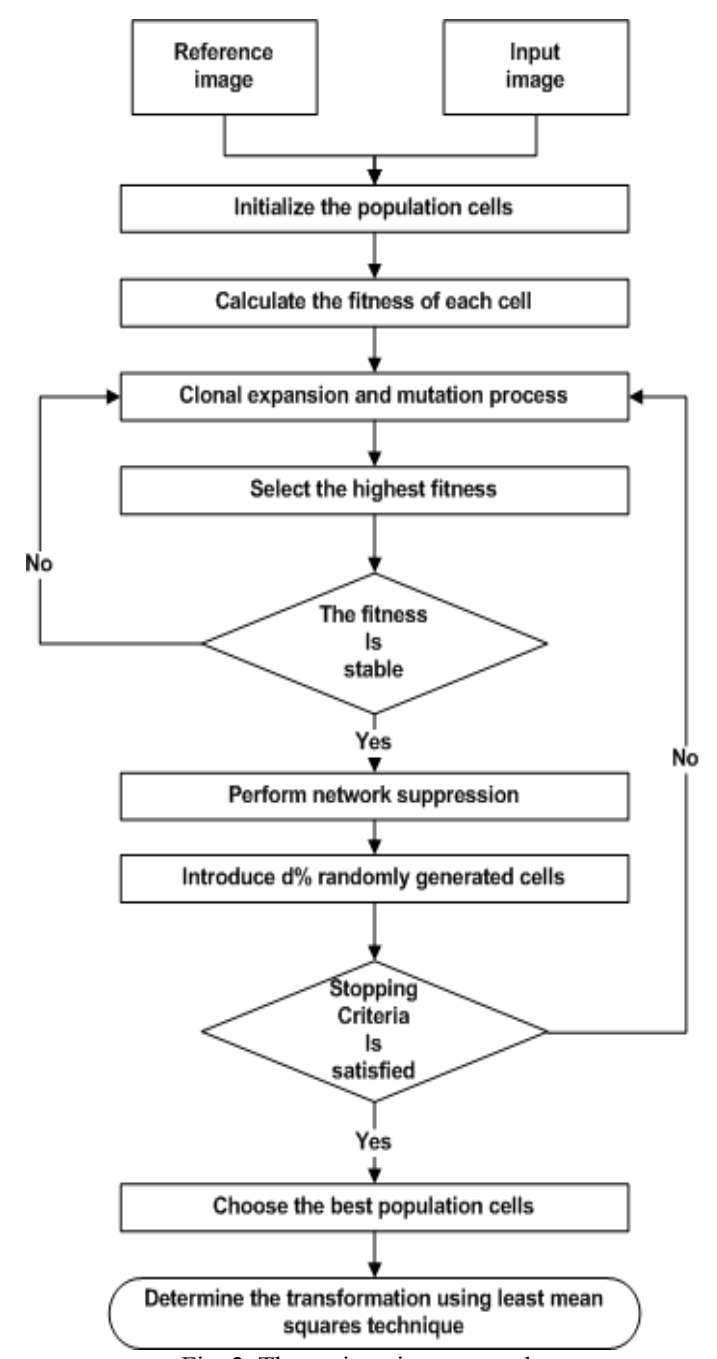

Fig. 2. The registration approach.

$$
\text { Population }=\left(\begin{array}{cccccc}
x_{1} & y_{1} & d x_{1} & d y_{1} & s_{1} & \theta_{1} \\
x_{2} & y_{2} & d x_{2} & d y_{2} & s_{2} & \theta_{2} \\
\cdot & \cdot & \cdot & \cdot & \cdot & \cdot \\
\cdot & \cdot & \cdot & \cdot & \cdot & \cdot \\
x_{n} & y_{n} & d x_{n} & d y_{n} & s_{n} & \theta_{n}
\end{array}\right)
$$

Fig. 3. The population arrangement

$$
\left[\begin{array}{l}
x^{-} \\
y^{-}
\end{array}\right]=\left[\begin{array}{cc}
s \cos (\theta) & -s \sin (\theta) \\
s \sin (\theta) & s \cos (\theta)
\end{array}\right]\left[\begin{array}{l}
x \\
y
\end{array}\right]+\left[\begin{array}{l}
d x \\
d y
\end{array}\right]
$$

3) The squared of normalized cross correlation coefficient is used as a fitness function, this fitness is calculated for each row from the population, this fitness function is normalized between the interval $[0,1]$ so that we can used it directly. The fitness function formula is described below in Eq. (3) where a1 is a square patch around each pixel in the population, the coordinates of a1 is warped using it's parameters $(\mathrm{dx}, \mathrm{dy}, \mathrm{S}$, $\theta$ ) to give a 2 which is the correspondence patch (around the correspondence pixel) in the input image, $\mathrm{m} 1$ and $\mathrm{m} 2$ is the 
mean of a1 and a 2 respectively.

$$
\text { fitness }=\frac{\left[\sum_{x, y}(a 1(x, y)-m 1)(a 2(x, y)-m 2)\right]^{2}}{\sum_{x, y}(a 1(x, y)-m 1)^{2} \sum_{x, y}(a 2(x, y)-m 2)^{2}}
$$

4) In this paper a modified mutation formula is proposed where Eq. (1), here the decay factor $\beta$ is eliminated and the mutation percentage is multiplied by the search range. Each cell component is mutated according to the proposed mutation formula described in Eq. (4)

$$
\begin{gathered}
c^{-}=c+\alpha \cdot N(0,1) \\
\alpha=\exp \left(-f^{*}\right) \cdot\left(S_{\text {max }}-S_{\text {min }}\right)
\end{gathered}
$$

where $C^{-}$is the mutated component, $c$ is the old value of the components, $f^{*}$ is the normalized fitness function, $S_{\max }$ is maximum value of the parameter, $S_{\min }$ is minimum value of the parameter. In the mutation stage; a number of $n_{c}$ clones is created for each cell then these clones are mutated, the clone has the highest fitness is selected, if this clone has a fitness bigger than the original population cell fitness then it replaced it. Each cell component is mutated with a constraint, that is the location of the new clone $(x, y)$ must be in the reference image coordinates and any clone exceed $S_{\max }$ or $S_{\text {mim }}$ is refused.

The process of clonal expansion and the mutation is repeated until the average fitness is stabilized and dropped below predefined threshold.

5) To prevent the correspondence points from concentrating in a small region in the image, a point suppression process is needed via keep a minimum distance among the points in the population, all points lie within this distance will be deleted from the population except the point which has the highest fitness.

6) Regeneration is needed after the suppression process so that, $\mathrm{d} \%$ fresh cells is inserted to the population, each cell has a value within the search range for each parameter $\left[S_{\text {min }}\right.$. $\left.S_{\text {max }}\right]$.

7) All the previous steps is repeated until the predefined number of iteration is reached; here the correspondence step is completed.

8) Finally, we select the population cells that have fitness higher than a predefined value, here excellent point correspondence between the reference and input images is established so the least mean squares technique [9] is used directly to find the global transformation between the reference and input images.

\section{EXPERIMENTS AND RESULT}

\section{A. Data set Description}

We used two sets of images to demonstrate the effectiveness of the proposed approach, including graphical image sequence (car sequence) and medical image sequence ( MRI sequence) each car image has a size 121x72 pixels,

also we used a medical images with a size 160x160 pixels. The initial number of $n_{\text {int }}$ was 60 , we used ten clones $\left(n_{c}=10\right)$ for each cell, $10 \times 10$ patch around each point in reference image, six pixel suppression threshold in x-direction is used, the regeneration percentage was $40 \%$, the average fitness stabilized when it drop below 0.001 , fitness less than 0.96 is removed from the population, finally a 100 iteration is used to perform this process. All the pervious parameters are tuned experimentally to get the better results.

We used the iterative closest point (ICP) algorithm [9]-[10] and random sample Consensus (RANSAC) algorithm [12] to compare our approach accuracy versus these algorithms accuracies. Both ICP and RANSAC are correspondence algorithms required features detector, so that the well-known Harris corners detector [13] is used to find some control points in the reference and input images. This approach is implemented using MATLAB programming language.

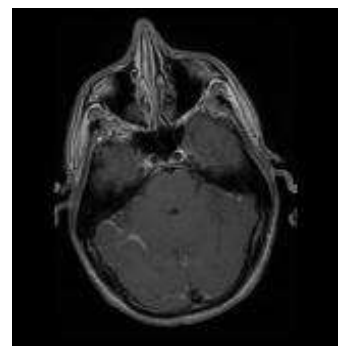

(a)

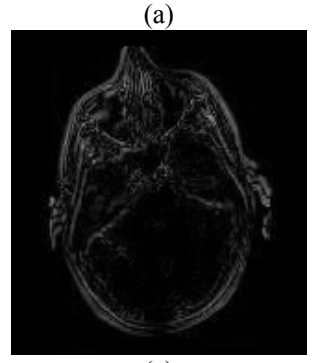

(c)

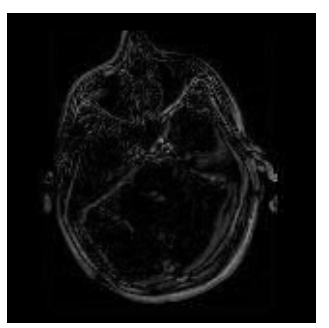

(e)

Fig. 4. MRI images, (a) reference image, (b) input image, the registration error in case of using; (c) proposed approach (d) ICP and (e) RANSAC.

\section{B. Visual and Comparative Results}

After the simulation, we have observed very good improvement through the use of our approach with no decrease in registration accuracy.

The first experiment is shown Fig. 4; where a medical images are used, the reference image in (a), the input image in (b), the proposed approach registration error in (c), the registration error in the case of ICP and RANSAC shown in (d), (e) respectively, it is clear that the proposed approach produce a small registration error compared to ICP and RANSAC. In Table 1 the average peak signal to noise ratio (PSNR) in dB over 10 runs is calculated. The PSNR is defined as: 


$$
\operatorname{PSNR}=\frac{f_{\text {max }}^{2}}{E\left[\left(f_{\text {ref }}-f_{\text {reg }}\right)^{2}\right]}
$$

where $f_{\max }$ is the maximum intensity of the image, $\mathrm{E}[\mathrm{]}$ is the ensemble average, $f_{\text {ref }}$ is the reference image intensity and $f_{\text {reg }}$ is the registered image intensity. The proposed approach has $45.8126 \mathrm{~dB}$ while the ICP algorithm has $45.6460 \mathrm{~dB}$ and $45.6853 \mathrm{~dB}$ for RANSAC algorithm. These refer to the improvement in the registration accuracy when our registration approach is used.
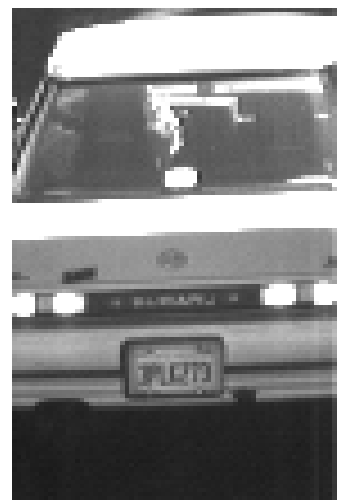

(a)

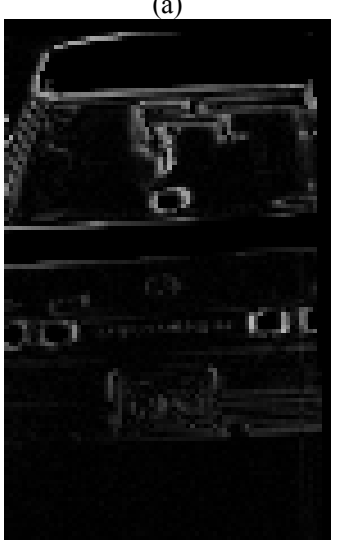

(c)

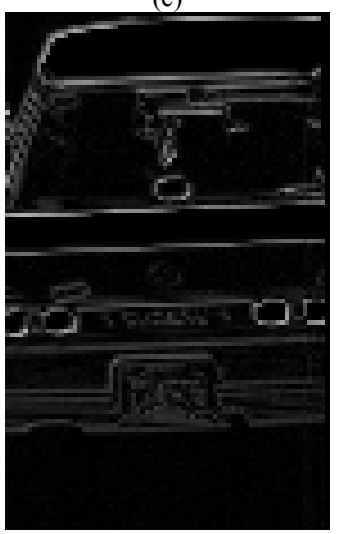

(e)

Fig. 5. Car images, (a) reference image, (b) input image, the registration error in case of using; (c) proposed approach, (d) ICP and (e) RANSAC.

The second experiment is shown in Fig. 5; where a car images are used, the reference image in (a), the input image in (b) and their registration error in(c), the registration error when ICP is used shown in (d) and the RANSAC algorithm registration error is shown in (e). In this case as it presented in table 1 the proposed approach has $46.1626 \mathrm{~dB}$ average PSNR while the PSNR of the ICP and RANSAC algorithms are $31.2637 \mathrm{~dB}, \quad 43.3182 \mathrm{~dB}$ respectively. These results demonstrate the effectiveness of the proposed approach over the ICP and RANSAC algorithms.

In Table II the main differences among the proposed algorithm, ICP and RANSAC algorithms has been summarized for different viewpoints.

TABLE I: THE AVERAGE PSNR COMPARISON.

\begin{tabular}{|l|l|l|l|}
\hline Data Set & $\begin{array}{l}\text { The proposed } \\
\text { approach }\end{array}$ & ICP & RANSAC \\
\hline $\begin{array}{l}\text { Medical } \\
\text { images }\end{array}$ & 45.8126 & 45.6460 & 45.6853 \\
\hline Car images & 46.1626 & 31.2637 & 43.3182 \\
\hline
\end{tabular}

TABLE II: COMPARISON.

\begin{tabular}{|l|l|l|l|}
\hline Viewpoints & $\begin{array}{l}\text { The proposed } \\
\text { approach }\end{array}$ & ICP & RANSAC \\
\hline $\begin{array}{l}\text { Feature } \\
\text { detector }\end{array}$ & Not needed & $\begin{array}{l}\text { Mandatory } \\
\text { step }\end{array}$ & $\begin{array}{l}\text { Mandatory } \\
\text { step }\end{array}$ \\
\hline $\begin{array}{l}\text { Warp } \\
\text { parameters } \\
\text { search range }\end{array}$ & Pre-Set & Not controlled & Not controlled \\
\hline $\begin{array}{l}\text { Performance } \\
\text { Max. No. of } \\
\text { iteration }\end{array}$ & 100 & $\begin{array}{l}\text { Depend on } \\
\text { The feature } \\
\text { extraction step }\end{array}$ & $\begin{array}{l}\text { Depend on } \\
\text { extraction step }\end{array}$ \\
\hline
\end{tabular}

\section{CONCLUSIONS}

In this paper we proposed a new approach for image registration, where AIS is used to determine some correspondence points between the reference and input images then the least mean squares technique is used to determine the global motion between the two images. The results show that the proposed approach gives a good accuracy compared to ICP and RANSAC algorithms in addition to our approach doesn't require any additional refinement or feature extraction as other point based approaches. The future works focusing on combining this registration approach with a segmentation process to improve the registration via determine the motion of each region in the image; also a higher order transformation model like projective transformation will be used.

\section{ACKNOWLEDGMENT}

The RANSAC algorithm we used for comparison has been implemented in MATLAB by Marco Zuliani, 2011.

\section{REFERENCES}

[1] S. Baker and I. Matthews, "Equivalence and efficiency of image alignment algorithms," in Proceedings of the IEEE Conference on Computer Vision and Pattern Recognition, vol. 1, pp. 1090-1097, 2001.

[2] M. Johansson, "Image Registration with Simulated Annealing and Genetic Algorithms," MSc dissertation, KTH computer science and communication, Stockholm, Sweden, 2006. 
[3] D. Deguchi, K. Mori, M. Feuerstein, T. Kitasaka, C. R. Maurer, Y. Suenaga, H. Takabatake, M. Mori, and H. Natori, "Selective image similarity measure for bronchoscope tracking based on image registration," Medical Image Analysis, vol. 13, no. 4, pp. 621-633, 2009.

[4] Z. Jankó, D. Chetverikov, and A. Ekárt, "Using a genetic algorithm to register an uncalibrated image pair to a 3D surface model," Engineering Applications of Artificial Intelligence, vol. 19, no. 3, pp. 269-276, 2006.

[5] F. L. Seixas, L. S. Ochi, A. Conci, and D. C. Saade, "Image registration using genetic algorithms," GECCO'08, 2008.

[6] K. K. Delibasis, P. A. Asvestas, and G. K. Matsopoulos, "Automatic point correspondence uses an artificial immune system optimization technique for medical image registration," computerized medical imaging and graphics, vol. 35, pp. 31-41, 2011.

[7] F. M. Ye, S. P. Xu, and Y. H. Xiong, "Two-step image registration by artificial immune system and chamfer matching," Chinese Optics Letters, vol. 6, no. 9, pp. 651-653, 2008.

[8] L. N. De Castro and J. Timmis, "An artificial immune network for multimodal function optimization," Congress on Evolutionary Computation (CEC'02), vol. 1, pp. 699-674, 2002.

[9] Lecture notes on image registeration. [Online]. Available: http://www.cs.rpi.edu/academics/courses/spring04/imagereg, 2011.

[10] P. J. Besl and N. D. McKay, "A method for registration of 3-D shapes," IEEE Trans. Patt. Anal. Mach. Intell., vol. 14, no. 2, pp. 239-256, 1992.
[11] A. A. Goshtasby, 2-D and 3-D Image Registration for medical, remote sensing and industrial applications, John Wiley \& Sons, Inc., 2005.

[12] M. A. Fischler and R. C. Bolles, "Random sample consensus: A paradigm for model fitting with applications to image analysis and automated cartography," Communications of the ACM, vol. 24, no. 6, pp. 381-395, 1981.

[13] C. Harris and M. Stephens, "A combined corner and edge detector," Alvey Vision Conference, pp. 147-151, 1988.

Osama A. Omer Received his B.Eng. and M. Eng. degrees in electrical engineering from South Valley University, Aswan, Egypt, in 2000 and 2004 respectively. He received his $\mathrm{Ph} . \mathrm{D}$. degree from Tokyo University of Agriculture and Technology in 2009. He is now assistant professor at Aswan University. He spent the summer internship 2008 in Nokia/Tokyo research center. His interests include image/video super resolution and image/video compression.

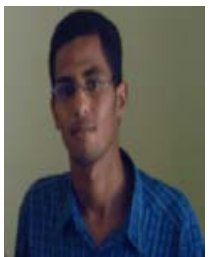

Mohamed Abdel-Nasser is an assistant lecturer at Electrical Engineering Department, Aswan University, Egypt. He has obtained the B.Sc. in communication and electronics from Aswan faculty of engineering, South Valley University, Egypt, in 2009. His interests include medical image registration, medical image super-resolution. 